ARTIGO ORIGINAL

\title{
Valores séricos para eletrólitos e minerais em jabutis Chelonoidis carbonaria (Reptilia, Testudinidae) mantidos em cativeiro no município de Uberlândia, MG
}

\author{
Blood profile for eletrolites and minerals in jabutis Chelonoidis carbonaria \\ (Reptilia, Testudinidae) kept in captivity in the city of Uberlândia, Brazil
}

\footnotetext{
Paulo Vinícius Rocha Pereira (D) ${ }^{1,2}$, Saulo Gonçalves Pereira (D) ${ }^{1,2}$, Adriele Laurinda Silva $\mathbb{( D}^{1,2}$, Eva Mendes Monteiro (D) ${ }^{1,2}$, Taciano dos Reis Cardoso (iD) ${ }^{1,2 *}$, André Luiz Quagliatto Santos (iD) ${ }^{2}$

${ }^{1}$ Faculdade Cidade Patos de Minas (FPM), Patos de Minas, MG, Brasil

${ }^{2}$ Universidade Federal de Uberlândia (UFU), Uberlândia, MG, Brasil
}

\section{Resumo}

Chelonoidis carbonaria - Spix, 1824, (jabuti-piranga ou jabuti-das-patas-vermelhas) é considerado o principal testudine mantido em cativeiro e possui amplo comércio ilegal por se tratar de uma espécie muito procurada como animal de estimação. 0 conhecimento acerca dos valores séricos metabólicos de $C$ carbonaria, portanto, é de grande relevância na prática clínica destes animais. Sendo assim, objetivou-se mensurar os valores dos constituintes bioquímicos minerais e dos eletrólitos séricos (cálcio, cloretos, ferro, fósforo, magnésio, potássio e sódio) em C. carbonaria mantidos em cativeiro. Para tanto, foram utilizados 25 animais jabuti-piranga, de ambos os sexos, dos quais foram coletadas amostras sanguíneas para análise bioquímica de minerais e eletrólitos séricos com analisador automático (Flexor XL). A análise estatística foi descritiva e inferencial, por meio do teste paramétrico T Studente do não paramétrico Wilcoxon, sendo considerado nível de significância de 5\% (p < 0,05). Os resultados evidenciaram que não houve diferenças estatísticas significativas dos valores séricos entre machos e fêmeas ( $p$ > 0,05), e também não demonstraram diferenças nas médias observadas em comparação a outras espécies de Testudines realizados por outros estudos. Conclui-se que os constituintes bioquímicos minerais e os eletrólitos séricos não demonstraram diferenças significativas entre os sexos de $C$. carbonaria e nem desta espécie com outras espécies de quelôneos.

Palavras-chave: Bioquímica sanguínea. Mineralograma. Perfil metabólico. Répteis. 


\section{Abstract}

The jabuti-piranga or red-legged tortoise (Chelonoidis carbonaria - Spix, 1824) is one of the most common testudines kept in captivity, and is often traded illegally as a popular pet. Therefore, knowledge about the metabolic serum values of C. carbonaria is particularly relevant in the clinical care of these animals. This study aimed to measure the values of the mineral biochemical constituents and serum electrolytes (calcium, chloride, iron, phosphorus, magnesium, potassium, and sodium) in captive C. carbonaria. For this purpose, blood samples were collected from 25 jabuti-piranga species, including animals of both sexes. Blood samples were submitted for biochemical analysis of minerals and serum electrolytes with an automatic analyzer (Flexor XL). Statistical analysis was descriptive and inferential, using the Student's t-test and a nonparametric Wilcoxon test. The results were considered significant when the p-value was less than 0.05. There were no statistically significant differences in the serum values between male and female $\mathrm{C}$. carbonaria ( $p$ > 0.05). Values in C. carbonaria were not significantly different from the means observed in other testudine species of previous studies. We concluded that the mineral biochemical constituents and the serum electrolytes of C. carbonaria are neither significantly different between sexes nor in comparison to the same values of other chelonian species.

Keywords: Blood Biochemistry. Mineral. Profile Metabolic. Reptiles.

\section{Introdução}

A espécie Chelonoidis carbonaria (Reptilia, Testudinidae), popularmente conhecida como jabuti-piranga ou jabuti-das-patas-vermelhas, está amplamente distribuída no Brasil e em outros países da América do Sul. É considerada o principal Testudine e o que mais tem sido mantido em cativeiro como animal de estimação, devido a seu amplo comércio ilegal, justificando, assim, mais estudos sobre tal espécie (Carvalho, 2004; Ramos, et al., 2009; Rodrigues, 2011).

Os processos vitais normais de todas as formas de vida requerem elementos bioquímicos para serem realizados. A ingestão equilibrada de minerais é essencial para evitar deficiências orgânicas. A matéria mineral corporal está em sua maioria (80 a $85 \%$ ) nos ossos e consiste principalmente de macrominerias como sais de cálcio $(\mathrm{Ca})$, fósforo $(\mathrm{P})$, magnésio $(\mathrm{Mg})$, sódio $(\mathrm{Na})$, cloro $(\mathrm{Cl})$, potássio $(\mathrm{K})$ e, em concentrações menores, de microminerais como iodo (I), zinco ( $\mathrm{Zn})$, ferro (Fe), entre outros (Gonzalez et al., 2000).

0 perfil sanguíneo pode fornecer informações do metabolismo de minerais e eletrólitos séricos, além de oferecer informações para interpretação do funcionamento fisiológico e dos processos (Souza et al., 2016). 0 conhecimento sobre dados bioquímicos é de grande importância para a sanidade e nutrição de espécies em cativeiro. Sendo assim, objetivouse mensurar as concentrações de minerais séricos em $C$. carbonaria de ambos os sexos, mantidos em cativeiro, utilizando métodos analíticos de rotina e acrescentando subsídios para o clínico. Por fim, objetivou-se comparar os dados com outros parâmetros publicados por outros autores para outras espécies.

Os resultados obtidos neste estudo podem contribuir para que haja um melhor entendimento dos parâmetros bioquímicos de minerais e eletrólitos séricos desses jabutis, e facilitar a interpretação dos mesmos. Podem, também, subsidiar outros estudos que busquem valores de referência para C. carbonaria.

\section{Material e métodos}

A pesquisa foi autorizada pelo Instituto Chico Mendes de Conservação da Biodiversidade (ICMBio), sob o $\mathrm{n}^{\circ} 46723-2$, e foi aprovada pela Comissão de Ética na Utilização de Animais (CEUA) da Universidade Federal de Uberlândia (UFU), conforme protocolo ${ }^{\circ}$ 035/15.

Foram utilizados 25 exemplares de $C$. carbonaria, sendo 15 machos e 10 fêmeas, oriundos de resgaste realizado pela Polícia Militar Ambiental ou entrega voluntária e pertencentes ao plantel do Laboratório de Pesquisa em Animais Silvestres (LAPAS), da Faculdade de Medicina Veterinária (FAMEV) da UFU, em Uberlândia, localizada na região sudoeste do estado de Minas Gerais. 
Os animais se encontravam alojados em recinto de alvenaria, com solário e local sombreado, densidade máxima de ocupação de 10 animais/4 $\mathrm{m}^{2}$, água clorada à vontade e alimentação fornecida diariamente, com verduras, frutas, legumes e ração comercial para cães, com no mínimo 18\% de proteína bruta.

Foram incluídos no grupo experimental apenas espécimes do plantel cujo comprimento curvilíneo da carapaça (CCC) fosse maior ou igual a $25 \mathrm{~cm}$, para que as características sexualmente dimórficas fossem vislumbradas a contento (Jerozolimski, 2005). Todos os animais foram avaliados fisicamente quanto à condição corporal, presença de ectoparasitas, tumores e lesões cutâneas. Foram selecionados 25 animais hígidos e sem deformidades de carapaça e/ou plastrão. A medida da carapaça foi aferida utilizando-se uma fita métrica flexível (precisão de 0,5 cm), aplicada sobre a carapaça dos animais e seguindo o seu contorno, desde a sutura entre os primeiros escudos marginais anteriores, esquerdo e direito, até o escudo supracaudal, conforme descrito por Montenegro (2004).

As características avaliadas para observação da dismorfia sexual foram: menor grau de convexidade da carapaça, concavidade e pigmentação mais acentuada do plastrão, apresentada pelos machos adultos em detrimento das fêmeas, que apresentam plastrão plano. 0 tamanho da cauda também foi observado, visto que a dos machos é maior e mais robusta que a das fêmeas (Levine e Schafer, 1992).

Os jabutis foram contidos manualmente para coleta de amostra sanguínea. Foram colhidos, aproximadamente, $4 \mathrm{ml}$ de sangue venoso diretamente da cauda ou das veias braquiais de cada animal, em seringa descartável de $5 \mathrm{~mL}$, com agulha hipodérmica descartável $27 \mathrm{G}$, após prévia antissepsia do local com álcool 70\%. 0 sangue foi transferido para tubos e estes foram centrifugados (centrífuga Excelsa Baby, FANEM, modelo 208N; 720 g), por 5 minutos, para obtenção do soro (Almosny e Monteiro, 2007), que foi separado e refrigerado na temperatura de 2 a $8{ }^{\circ} \mathrm{C}$, por no máximo 8 horas, até as análises. Em seguida, as amostras foram encaminhadas em caixas isotérmicas com gelo para o Laboratório de Análises Clínicas da Faculdade Patos de Minas, em Patos de Minas, MG, onde foram processadas. As amostras de soro hemolisadas não foram utilizadas nas análises.
As análises bioquímicas de minerais e eletrólitos séricos (Tabela 1) foram realizadas em analisador automático FLEXOR XL (Vital Scientific/Elitch), usando reagentes da marca Elitech Clinical Systems SAS, segundo recomendações do fabricante, à temperatura de $37^{\circ} \mathrm{C}$, após prévia calibração do equipamento com calibrador multiparamétrico (Elitech Elical II) e aferimento com soro controle (Control Lab). Determinou-se, de cada amostra, os valores séricos dos minerais e eletrólitos. As respectivas metodologias empregadas estão descritas na Tabela 1.

Tabela 1 - Constituintes minerais séricos avaliados e respectivas metodologias empregadas para análises bioquímicas em Chelonoidis carbonaria

\begin{tabular}{ll}
\hline Constituinte bioquimico & Metodologia \\
\hline Cálcio total $(\mathrm{Ca})$ & Arsenazo III \\
Cloretos $(\mathrm{Cl})$ & Potenciometria/Eletrodo seletivo \\
Ferro $(\mathrm{Fe})$ & Cromazurol B \\
Fósforo $(\mathrm{P})$ & Molibdato UV \\
Magnésio $(\mathrm{Mg})$ & Colorimetria-Calmagite \\
Potássio $(\mathrm{K})$ & Potenciometria/Eletrodo seletivo \\
Sódio $(\mathrm{Na})$ & Potenciometria/Eletrodo seletivo \\
\hline
\end{tabular}

Realizou-se análise exploratória dos dados considerando todos os animais e cada sexo, de acordo com o proposto por Vieira (1981) e Ayres et al. (2007), fazendo uso do programa BioEstat (Ayres et al., 2007) e do aplicativo Action (Portal Action ${ }^{\circledR}$ 2015), que utiliza o programa R (R Core Team, 2015).

\section{Resultados e discussão}

Os resultados obtidos para minerais e eletrólitos séricos estão dispostos na Tabela 2 . Os testes de comparação entre os valores encontrados nos machos e fêmeas para minerais e eletrólitos séricos apresentaram $\mathrm{p}>0,05$, portanto, não há evidências de diferenças significativas entre os dois grupos, estabelecendo-se, assim, intervalo de confiança único para minerais e eletrólitos séricos. 
Tabela 2 - Médias, desvios padrão, amplitudes de variação e intervalos de confiança de 95\% para a média populacional dos minerais e eletrólitos séricos de Chelonoidis carbonaria ( $\mathrm{n}=$ 25), mantidos em cativeiro em Uberlândia, MG, 2015

\begin{tabular}{cccccccc}
\hline & & & & \multicolumn{2}{c}{ Amplitude de variação } & \multicolumn{2}{c}{ IC 95\% } \\
\cline { 7 - 8 } $\begin{array}{c}\text { Constituintes minerais } \\
\text { e eletrolíticos }\end{array}$ & Unidade & Média & Desvio padrão & Mínimo & Máximo & $\begin{array}{c}\text { Limite } \\
\text { inferior }\end{array}$ & $\begin{array}{c}\text { Limite } \\
\text { superior }\end{array}$ \\
\hline Cálcio total $(\mathrm{Ca})$ & $\mathrm{mg} / \mathrm{dL}$ & 9,02 & 1,83 & 6,20 & 12,60 & 8,22 & 9,84 \\
Ferro $(\mathrm{Fe})$ & $\mu \mathrm{g} / \mathrm{dL}$ & 46,05 & 17,21 & 10,00 & 75,00 & 38,42 & 53,69 \\
Fósforo $(\mathrm{P})$ & $\mathrm{mg} / \mathrm{dL}$ & 2,14 & 0,92 & 0,90 & 4,30 & 1,76 & 2,52 \\
Magnésio $(\mathrm{Mg})$ & $\mathrm{mg} / \mathrm{dL}$ & 4,70 & 0,93 & 3,48 & 6,73 & 4,35 & 5,16 \\
Cloretos $(\mathrm{Cl})$ & $\mathrm{mEq} / \mathrm{L}$ & 90,58 & 4,77 & 81,00 & 101,00 & 88,56 & 92,59 \\
Potássio $(\mathrm{K})$ & $\mathrm{mEq} / \mathrm{L}$ & 3,21 & 0,70 & 1,90 & 4,70 & 2,92 & 3,50 \\
Sódio $(\mathrm{Na})$ & $\mathrm{mEg} / \mathrm{L}$ & 113,93 & 8,14 & 97,00 & 130,00 & 110,52 & 117,39 \\
\hline
\end{tabular}

Para os minerais e eletrólitos séricos (Tabela 3), os níveis de Ca total encontrados para C. carbonaria em cativeiro $[9,02( \pm 1,83) \mathrm{mg} / \mathrm{dL}]$ são próximos aos descritos por Ferreira $(2002)[10,79( \pm 1,36) \mathrm{mg} /$ dL] em população de $C$. carbonaria e $C$. denticulata, e ligeiramente inferiores às médias descritas por Carpenter (2005) tanto para C. carbonaria $[12,6( \pm$ $2,3) \mathrm{mg} / \mathrm{dL}]$, quanto para $C$. elegans $[11,6( \pm 3,0)$ $\mathrm{mg} / \mathrm{dL}]$ e $C$. radiata $[12,2(10,8-14,4) \mathrm{mg} / \mathrm{dL}]$.

Valores similares foram descritos em Geochelone gigantea (jabutis-gigantes) por Ghebremeskel et al. (1991) $(9,3 \pm 2,5 \mathrm{mg} / \mathrm{dL})$, e em Chelonia mydas (tartaruga-verde) pelos autores Bolten e Bjorndal (1992) $(9,1 \pm 2,1 \mathrm{mg} / \mathrm{dL})$ e Fong et al. (2010) $[8,87$
$( \pm 1,62) \mathrm{mg} / \mathrm{dL}]$. Médias de Ca sérico ligeiramente inferiores foram encontradas por Santos et. al. (2005) $[7,08( \pm 1,04) \mathrm{mg} / \mathrm{dL}]$ e por Mundim et al. (2003) e Fonseca et al. (2016) $[8,03( \pm 0,85) \mathrm{mg} /$ $\mathrm{dL}]$, ambas em população de Podocnemis expansa mantidas em cativeiro.

Vale ressaltar que os níveis de Ca total encontrados no presente estudo para $C$. carbonaria estão dentro dos valores descritos para répteis, entre 8 a $11 \mathrm{mg} / \mathrm{dL}$. Os valores de Ca em répteis, entretanto, devem ser interpretados levando em consideração suas particularidades fisiológicas; em espécies ovíparas, por exemplo, há importantes elevações durante a vitelogênese.

Tabela 3 - Médias por sexo, testes estatísticos e p-valor de minerais e eletrólitos séricos de Chelonoidis carbonaria $(n=25)$, mantidas em cativeiro em Uberlândia, MG, 2015

\begin{tabular}{|c|c|c|c|c|}
\hline \multirow[b]{2}{*}{ Constituinte bioquímico } & \multicolumn{2}{|c|}{ Media por sexo } & \multirow[b]{2}{*}{ Teste } & \multirow[b]{2}{*}{ p-valor } \\
\hline & Macho & Fêmea & & \\
\hline Cálcio (Ca) (mg/dL) & 9,02 & 9,05 & Wilcoxon & 0,637 \\
\hline Ferro $(\mathrm{Fe})(\mu \mathrm{g} / \mathrm{dL})$ & 46,0 & 46,10 & Wilcoxon & 1 \\
\hline Fósforo (P) (mg/dL) & 2,15 & 2,13 & Wilcoxon & 1 \\
\hline Magnésio (Mg) (mg/dL) & 5,65 & 3,70 & Wilcoxon & 0,338 \\
\hline Cloretos (Cl) (mEq/L) & 89,80 & 91,25 & $\mathrm{t}$-Student & 0,754 \\
\hline Potássio (K) (mEq/L) & 3,01 & 3,41 & $\mathrm{t}$-Student & 0,469 \\
\hline Sódio (Na) (mEq/L) & 112,25 & 115,60 & $\mathrm{t}$-Student & 0,528 \\
\hline
\end{tabular}


Os valores médios da concentração sérica de $\mathrm{P}$ em C. carbonaria, de 2,14 $( \pm 0,92) \mathrm{mg} / \mathrm{dL}$, encontramse dentro dos valores de referência para répteis $(1,0$ a $5,0 \mathrm{mg} / \mathrm{dL}$ ) relatados por Martínez-Silvestre et al. (2013), porém inferiores aos valores em $C$. elegans $[3,8( \pm 1,0) \mathrm{mg} / \mathrm{dL}]$, C. radiata $[3,2(2,6 \mathrm{~m}-4,3) \mathrm{mg} /$ $\mathrm{dL}]$ e $C$. carbonaria $[3,6( \pm 1,2) \mathrm{mg} / \mathrm{dL}]$ encontrados por Carpenter (2005).

Ao comparar os valores de $\mathrm{P}$ com aqueles descritos por Ferreira (2002) em C. carbonaria e C. denticulata $[4,67( \pm 2,13) \mathrm{mg} / \mathrm{dL}]$, por Samour et al. (1986) em Geochelone spp. $(4,34 \pm 3,9 \mathrm{mg} /$ $\mathrm{dL}$ ), por Bolten e Bjorndal (1992) em Chelonia mydas $[6,7 \pm 1,2 \mathrm{mg} / \mathrm{dL}]$, e por Santos et. al. (2005) $[5,21( \pm 1,22) \mathrm{mg} / \mathrm{dL}]$ e Mundim et. al. (2003) $[4,81( \pm 1,17) \mathrm{mg} / \mathrm{dL}]$ em Podocnemis expansa, observaram-se valores superiores aos encontrados na presente pesquisa.

Relata-se, em parte da literatura, algumas referências da influência do sexo nas concentrações séricas de Ca total e P. Diferenças estatisticamente significativas $(p<0,05)$, com valores mais elevados nas fêmeas, foram descritas por Ferreira (2002) em população de $C$. carbonaria e $C$. denticulata, por Raphael et al. (1994) em Malacochersus tornierie e por Christopher (1999) para jabutis Gopherus agassizii. Contudo, não corroboram o presente trabalho, visto que na população de $C$. carbonaria mantida em cativeiro não houve diferenças estatisticamente significativas para $\mathrm{Ca}$ e $\mathrm{P}$ entre machos e fêmeas (Tabela 3).

Os valores séricos de $\mathrm{Mg}$ obtidos para $C$. carbonaria foram de $4,70( \pm 0,93) \mathrm{mg} / \mathrm{dL}$. Ferreira (2002), em estudo com jabutis $C$. carbonaria e $C$. denticulata, descreveu média de $3,36( \pm 0,43) \mathrm{mg} /$ $\mathrm{dL}$, ligeiramente inferior às descritas no presente trabalho. Valores de Mg inferiores foram descritos também por Mundim et al. (2003) e Santos et al. (2005), ao avaliarem constituintes bioquímicos sanguíneos de Podocnemis expansa (tartaruga-daAmazônia) cativas, tendo sido relatadas médias para Mg de 1,45 $( \pm 0,17) \mathrm{mg} / \mathrm{dL}$ e de $1,60( \pm 0,14)$ $\mathrm{mg} / \mathrm{dL}$, respectivamente. Fong et al. (2010), em tartarugas Chelonia mydas, encontraram médias de 4,39 ( $\pm 2,28) \mathrm{mg} / \mathrm{dL}$; essas médias são bem próximas às descritas para $\mathrm{Mg}$ em $C$. carbonaria, todavia, convém salientar que no caso de $C$. Mydas o habitat é marinho.
As concentrações séricas de $\mathrm{Mg}$ podem estar elevadas em estados de desidratação e diminuídas em casos de má nutrição, pancreatite e distúrbios na glândula tireoide (Thrall et al., 2006). Para répteis, no entanto, hipomagnesemia é inabitual, mas tem sido proposta como coadjuvante de doenças ósseas e metabólicas por ser um cofator importante no metabolismo do cálcio (Campbell, 2006). Todavia, de acordo com Martínez-Silvestre et al. (2013), a aplicação clínica dos valores séricos de Mg para o diagnóstico de doenças ósseas é ainda incipiente, não havendo estudos suficientes que corroborem essa aplicação clínica.

Com relação ao $\mathrm{Fe}$, os valores encontrados para C. carbonaria $[46,05( \pm 17,21) \mu \mathrm{g} / \mathrm{dL}]$ são inferiores às médias relatadas por Ferreira (2002) $[75,21( \pm$ 17,02) $\mu \mathrm{g} / \mathrm{dL}$ ] em $C$. carbonaria e $C$. denticulata. Os valores encontrados neste trabalho foram discrepantes daqueles obtidos por Santos et al. (2005) $[390,35( \pm 116,65) \mu \mathrm{g} / \mathrm{dL}]$ e Mundim et al. (2003) $[471,04( \pm 301,80) \mu \mathrm{g} / \mathrm{dL}]$ em Podocnemis expansa, por Samour et al. (1986) $(162,01 \pm 128,49$ $\mu \mathrm{g} / \mathrm{dL})$ em Geochelone spp. em cativeiro e por Brites (2002) para Phrynops geoffroanus (176,86 a $369,50 \mu \mathrm{g} / \mathrm{dL}$ ).

Para os eletrólitos séricos em C. carbonaria, os valores encontrados para cloretos, potássio e sódio foram de 90,58 $( \pm 4,77) \mathrm{mEq} / \mathrm{L}, 3,21( \pm 0,70)$ $\mathrm{mEq} / \mathrm{L}$ e $113,95( \pm 8,14) \mathrm{mEq} / \mathrm{L}$, respectivamente (Tabela 2).

A hipocloremia sugere perda de íons de $\mathrm{Cl}$ ou carência de hidratação com líquidos contendo estes íons. Já a hipercloremia pode estar relacionada à desidratação ou a possíveis falhas renais (MartínezSilvestre et al., 2013).

As concentrações de Cl em C. carbonaria, de 90,58 $( \pm 4,77) \mathrm{mEq} / \mathrm{L}$, tiveram valores discretamente inferiores ao intervalo descrito para soro ou plasma de répteis por Martínez-Silvestre et al. (2013) (100 a $130 \mathrm{mEq} / \mathrm{L}$ ); entretanto, a média encontrada corrobora o valor encontrado por Carpenter (2005) para a espécie [98 $( \pm 5) \mathrm{mEq} / \mathrm{L}]$.

Valores similares de $\mathrm{Cl}$ foram registrados para Testudines oriundos de cativeiro por Samour et al. (1986) em Geochelone spp. [93,0 $( \pm 6,0) \mathrm{mEq} / \mathrm{L}]$ e por Santos et al. (2005) em Podocnemis expansa $[86,40 \quad \pm 7,63) \quad \mathrm{mEq} / \mathrm{L}]$. Médias superiores foram apresentadas por Ferreira (2002) para 
C. carbonaria e C. denticulata $[106,53$ ( $\pm 14,26)$ $\mathrm{mEq} / \mathrm{L}]$, por Bolten e Bjorndal (1992) [113,0 ( \pm $5,0) \mathrm{mEq} / \mathrm{L}]$ em Chelonia mydas e por Taylor Jr e Jacobson (1992) em Gopherus polyphemus [102,3 $( \pm 4,85) \mathrm{mEq} / \mathrm{L}]$.

Os répteis apresentam boa eficiência renal para eliminação de $\mathrm{K}$ e os seus valores séricos ou plasmáticos para répteis em geral estão entre 2 a 8 mEq/L (Martínez-Silvestre et al., 2013).

$O$ valor de K para C. carbonaria obtido no presente estudo $[3,21( \pm 0,70) \mathrm{mEq} / \mathrm{L}]$ está contido neste intervalo e próximo ao encontrado por Ferreira (2002) em $C$. carbonaria e $C$. denticulata mantidos em cativeiro $[2,74( \pm 0,93) \mathrm{mEq} / \mathrm{L}]$. Carpenter (2005) apresentou médias de 5,3 $( \pm 0,8) \mathrm{mEq} / \mathrm{L}$ para C. carbonaria, $5,2( \pm 0,7) \mathrm{mEq} / \mathrm{L}$ para C. elegans e 5,5 $(5,1-5,8) \mathrm{mEq} / \mathrm{L}$ para $C$. radiata. Médias superiores foram descritas também para outras espécies de Testudines por Taylor Jr. e Jacobson (1992) em Gopherus polyphemus $[5,01( \pm 0,32) \mathrm{mEq} / \mathrm{L}]$, por Raphael et al. (1994) em Malacochersus tornieri $[5,2( \pm 0,55) \mathrm{mEq} / \mathrm{L}]$ e por Samour et al. (1986) em Geochelone spp. $[4,7( \pm 1,0) \mathrm{mEq} / \mathrm{L}]$.

Segundo Selleri e Hernandez-Divers (2006), a ingestão inadequada de $\mathrm{K}$ ou perda do íon por processos diarreicos persistentes, enterites e patologias ou insuficiência renais podem proporcionar hipopotassemia em répteis. A hiperpotassemia pode se apresentar em animais com insuficiência renal em casos graves da doença, assim como em casos de hemólise da amostra sanguínea avaliada (Martínez-Silvestre et al., 2013). Raphael et al. (1994), com estudos em Malacochersus tornieri, afirmam que os níveis de $\mathrm{K}$ podem variar dependendo da dieta do animal.

Ao avaliar as concentrações de $\mathrm{Na}$ em $C$. carbonaria, de 113,95 $( \pm 8,14) \mathrm{mEq} / \mathrm{L}$, observase que Ferreira (2002) relatou valores próximos para essa variável $[100,04( \pm 22,11) \mathrm{mEq} / \mathrm{L}] \mathrm{em}$ população de jabutis $C$. carbonaria e $C$. denticulata. Entretanto, Carpenter (2005) apresenta resultados de Na ligeiramente superiores tanto para $C$. carbonaria $[128( \pm 5) \mathrm{mEq} / \mathrm{L}]$ quanto para outros jabutis do gênero Chelonoides: C. elegans $[131$ ( \pm 7) $\mathrm{mEq} / \mathrm{L}]$ e $C$. radiata [127 (121 - 132) $\mathrm{mEq} / \mathrm{L}]$. Valores superiores dessa variável foram descritos por Samour et al. (1986) em Geochelone spp. (tartaruga gigante) em cativeiro [132,5 $( \pm 2,5)$
$\mathrm{mEq} / \mathrm{L}]$, e Rosskopf Jr (1982) descreveu intervalo de 130 a 157 mEq/L em Gopherus agassizzi (jabutido-deserto californiano). Martínez-Silvestre et al. (2013) sugerem como referência um intervalo de 120 a $170 \mathrm{mEq} / \mathrm{L}$ para o $\mathrm{Na}$ em répteis no geral, estando os valores deste trabalho abaixo desse intervalo.

A hipernatremia em répteis pode ser observada em doenças renais, ingestão demasiada de $\mathrm{Na}$, desidratação, entre outros. A hiponatremia pode ser ocasionada por perdas excessivas de $\mathrm{Na}$, que associa-se a problemas gastrointestinais ou renais; em répteis, a hiponatremia pode ser decorrente da hipersecreção das glândulas de sal (McArthur, 2001).

\section{Conclusão}

Chelonoidis carbonaria não apresentam significativas diferenças de valores séricos de eletrólitos e minerais observados em outras espécies de Testudines. Não houve diferenças significativas entre as médias de machos e fêmeas para os parâmetros séricos estudados. Os dados aqui apresentados corroboram os descritos por outros autores para outras espécies de quelônios.

\section{Referências}

Almosny NRP, Monteiro AM. Patologia clínica. In: Cubas ZS, Silva JCR, Catão-Dias JL (EE.). Tratado de Animais Selvagens: medicina veterinária. São Paulo: Roca; 2007. p. 939-66.

Ayres M. BioEstat 5.0: aplicações estatísticas nas áreas das ciências biológicas e médicas. Belém: Sociedade Civil Mamirauá; 2007. 364 p.

Bolten AB, Bjorndal KA. Blood profiles for a wild population of green turtles (Chelonia mydas) in the southern Bahamas: size-specific and sex-specific relationships. J Wildl Dis. 1992;28(3):407-13.

Brites VLC. Hematologia, bioquímica do sangue, parasitologia, microbiologia, algas epizoárias e histopatologia de Phrynops geoffroanus (Schweigger, 
1812) (Testudinata, Chelidae), expostos a diferentes influências antrópicas no rio Uberabinha, Minas Gerais [tese]. São Carlos: Universidade Federal de São Carlos; 2002. 196 p.

Carpenter JW. Exotic animal formulary. Philadelphia: Saunders; 2005. 421 p.

Campbell TW. Bioquímica clínica de répteis. In: Thrall MA. Hematologia e bioquímica clínica veterinária. São Paulo: Roca; 2006. p. 461-6.

Carvalho RC. Topografia vértebro-medular e anestesia espinhal em jabuti das "patas vermelhas" Geochelone carbonaria (SPIX, 1824) [dissertação]. São Paulo: Universidade de São Paulo; 2004. 126 p.

Christopher MM. Physical and biochemical abnormalities associated with prolonged entrapment in a desert tortoise. J Wildl Dis. 1999;35(2):361-6.

Ferreira AB. Perfil bioquímico sanguíneo de jabutis (Geochelone carbonaria e Geochelone denticulata) [dissertação]. Uberlândia: Universidade Federal de Uberlândia; 2002.91 p.

Fonseca LA, Maia NL, Girardi FM, Carvalho Filho WP, Pimentel FG, Braga FR. Avaliação de parâmetros bioquímicos séricos em Tartarugas-da-Amazônia (Podocnemis expansa) mantidas em cativeiro. Pesq Vet Bras. 2016;36(12):1186-9.

Fong CL, Chen HC, Cheng IJ. Blood profiles from wild populations of green sea turtles in Taiwan. J Vet Med Anim Health. 2010;2(2):8-10.

Ghebremeskel K, Williams G, Spratt D, Samour HJ. Plasma biochemistry of free-living giant tortoise (Geochelone gigantea) on Curieuse Island (Republic of Seycheles). Comp Biochem Physiol A Physiol. 1991;99(1-2):65-7.

Gonzalez FHD, Barcelos JO, Patiño HO, Ribeiro LA. Perfil metabólico em ruminantes: seu uso em nutrição e doenças nutricionais. Porto Alegre: UFRGS; 2000.

Jerozolimski A. Ecologia de populações silvestres dos jabutis Geochelone denticulata e G. carbonaria (Cryptodira:
Testudinidae) no território da aldeia A'Ukresul do Pará [tese] São Paulo: Universidade de São Paulo; 2005. 155 p.

Levine D, Schafer D. Red-Footed Tortoise, Geochelone carbonaria. Tortuga Gaz. 1992;28(1):1-3.

Martínez-Silvestre A, González SL, Valera RC. La bioquímica sanguínea en clínica de reptiles. Cons Dif Vet. 2013;21(200):31-40.

McArthur S. Renal function in chelonians: dehydration and the stabilization of posthibernation hyperuricemia, hyperkalemia, and anuria in Testudo spp. Proc Assoc Reptilian Amphibian Veterinarians. 2001;8(1):87-96.

Montenegro PFGP. Efeitos do estresse e do cloridrato de quetamina sobre o padrão eletrocardiográfico, frequência cardíaca e comportamento de jabutis-piranga (Geochelone carbonaria - Spix, 1824) [dissertação] Recife: Universidade Federal de Pernambuco; 2004. 197 p.

Mundim AV, Queiroz RP, Santos ALQ, Beletti ME, Luz VLF. Bioquímica sanguínea da Tartaruga da Amazônia (Podocnemis expansa) em seu habitat natural. Biosc J. 1999;15(2):35-43.

Raphael BL, Klemens MW, Moehlman P, Dierenfeld E, Karesh WB. Blood values in free-ranging Pancake tortoises (Malacochersus tornieri). J Zoo Wildl Med. 1994;25(1):63-7.

Ramos RM, Vale DF, Hanawo MEOC, Ferreira FS, Luz MJ, Oliveira ALA. Penectomia em caso de prolapso peniano em Jabuti-piranga (Geochelone carbonaria): relato de caso. J Bras Cienc Anim. 2009; 2(3):166-74.

R Core Team. R: A language and environment for statistical computing. Vienna: R Foundation for Statistical Computing; 2015.

Rodrigues SS. Avaliação coproparasitológica de Chelonoidis carbonaria (Spix,1824) (Reptilia, Testudinidae) em cativeiro no Espírito Santo [dissertação]. Vila Velha: Centro Universitário Vila Velha; 2011. 101 p.

Rosskopf Jr WJ. Normal hemogram and blood chemistry values for California desert tortoises. Vet Med Small Anim Clin. 1982;77(1):85-7. 
Santos ALQ, Malta TS, Mundim AV, Alves Jr JRF, Carvalho SFM. Variação dos constituintes bioquímicos sanguíneos de tartarugas-da-amazônia (Podocnemis expansa, Schweigger - 1812) (testudinata) mantidas em criatório comercial. Arch Vet Sci. 2005;10(3):1-8.

Samour HJ, Hawkey CM, Pugsley S, Ball D. Clinical and pathological findings related to malnutrition and husbandry in captive giant tortoises (Geochelone species). Vet Rec. 1986;118(11):299-302.

Selleri P, Hernandez-Divers SJ. Renal diseases of reptiles. Vet Clin North Am Exot Anim Pract. 2006;9(1):161-74.

Souza BC, Sena LS, Loureiro D, Raynal JT, Souza TJ, Bastos $\mathrm{BL}$, et al. Determinação de valores de referência séricos para os eletrólitos magnésio, cloretos, cálcio e fósforo em ovinos das raças Dorper e Santa Inês. Pesq Vet Bras. 2016;36(3):167-73.
Taylor Jr RW, Jacobson ER. Hematology and serum chemistry of the gopher tortoise, Gopherus polyphemus. CompBiochem Physiol AComp Physiol. 1982;72(2):425-8.

Thrall MA, Weiser G, Allison RW, Campbell TW. Hematologia e bioquímica clínica veterinária. São Paulo: Roca; 2006. 450 p.

Vieira S. Introdução à bioestatística. Rio de Janeiro: Campus; 1981.120 p. 